\title{
A Peer Review of the Strategy for Characterizing Transuranics and Technetium Contamination in Depleted Uranium Hexafluoride Tails Cylinders
}

G.P. Brumburgh, H.I. Avci, M.J. Steindler, D.L. Bowers, S.K. Sengupta, E. Randich

\section{September 1, 2000}

U.S. Department of Energy

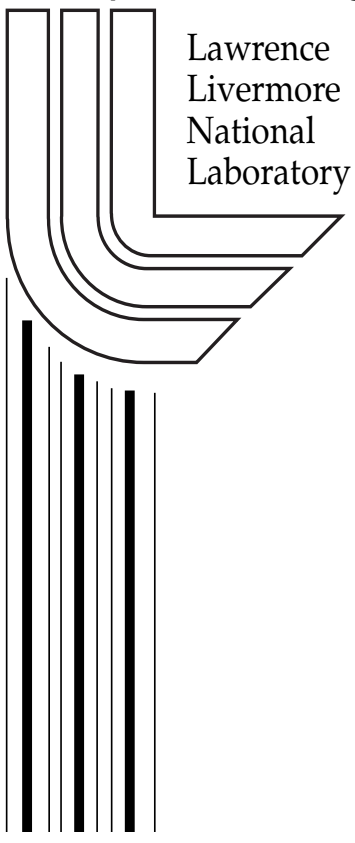




\section{DISCLAIMER}

This document was prepared as an account of work sponsored by an agency of the United States Government. Neither the United States Government nor the University of California nor any of their employees, makes any warranty, express or implied, or assumes any legal liability or responsibility for the accuracy, completeness, or usefulness of any information, apparatus, product, or process disclosed, or represents that its use would not infringe privately owned rights. Reference herein to any specific commercial product, process, or service by trade name, trademark, manufacturer, or otherwise, does not necessarily constitute or imply its endorsement, recommendation, or favoring by the United States Government or the University of California. The views and opinions of authors expressed herein do not necessarily state or reflect those of the United States Government or the University of California, and shall not be used for advertising or product endorsement purposes.

Work performed under the auspices of the U. S. Department of Energy by the University of California Lawrence Livermore National Laboratory under Contract W-7405-Eng-48.

This report has been reproduced directly from the best available copy.

Available to DOE and DOE contractors from the

Office of Scientific and Technical Information

P.O. Box 62, Oak Ridge, TN 37831

Prices available from (423) 576-8401

http://apollo.osti.gov/bridge/

Available to the public from the National Technical Information Service

U.S. Department of Commerce 5285 Port Royal Rd., Springfield, VA 22161 http://www.ntis.gov/

OR Lawrence Livermore National Laboratory Technical Information Department's Digital Library http://www.llnl.gov/tid/Library.html 
UCRL-ID-140343

\section{A Peer Review of the Strategy for Characterizing Transuranics and Technetium Contamination in Depleted Uranium Hexafluoride Tails Cylinders}

G.P. Brumburgh, Project Lead

Lawrence Livermore National Laboratory

H.I. Avci, M.J. Steindler, D.L. Bowers

Argonne National Laboratory

S.K. Sengupta, E. Randich

Lawrence Livermore National Laboratory 


\section{Table of Contents}

Executive Summary ……………………………….............................................

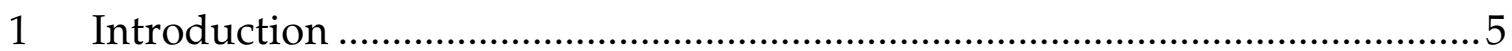

2 Purpose

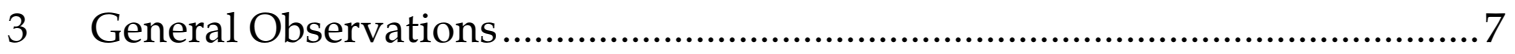

3.1 Transuranic Chemistry/Radioactivity ……………………………………………... 7

3.1.1 Observations: Americium Contamination in Tails Cylinders ..............................

3.1.2 Observations: Plutonium Contamination in Tails Cylinders................................8

3.1.3 Observations: Neptunium Contamination in Tails Cylinders..............................

3.1.4 Observations: Technetium Contamination in Tails Cylinders ..........................10

3.1.5 Observations: Fission Product Contamination in Tails Cylinders....................10

3.2 Statistical Approach Observations ……………………………………………..... 11

3.3 Uranium Enrichment Process................................................................................ 12

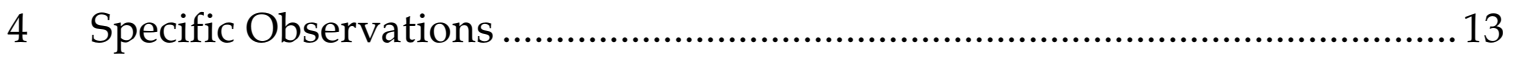

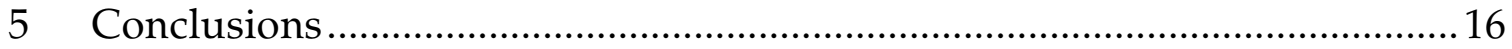

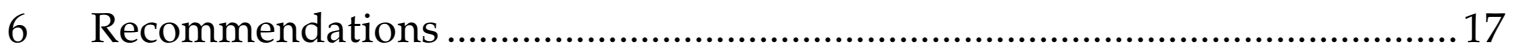

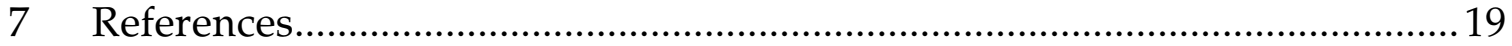

Appendix A: Hightower 2000 Study Conclusions ...............................................

Appendix B: Initial Q\&A Regarding the Review of the "Strategy for Characterizing Transuranics and Tc Contamination in UF $_{6}$ Tails Cylinders" .... B-1

Appendix C: History of Tails Cylinder Number 137128 ......................................... 


\section{Executive Summary}

This document provides the findings from a peer review of the Oak Ridge National Laboratory (ORNL) Hightower 2000 study, which set forth a strategy for characterization of low levels of radioactive contaminants in depleted uranium hexafluoride $\left(\mathrm{DUF}_{6}\right.$ ) cylinders at the Oak Ridge, Paducah, and Portsmouth gaseous diffusion plants. The information from that study will be used in a DOE-issued request for proposal (RFP) for conversion services from the private sector, to assist vendors in reducing costs associated with contingencies relating to handling this $\mathrm{DUF}_{6}$ with elevated transuranics (TRUs) and technetium (Tc).

The Hightower study developed a general strategy for DUF $_{6}$ sampling and analysis in accordance with their task but also recommended the following courses of action:

a) Collect and analyze liquid samples from the two identified Paducah Gaseous Diffusion Plant (PGDP) feed cylinders, feed the $\mathrm{DUF}_{6}$ into the cascades, and conduct an analysis on the resulting heels by washing and analyzing the wash rinsate

b) Characterize the cylinder coatings to determine the extent and levels of polychlorinated biphenyl (PCB) contamination

c) Verify the characterization of the cylinder inventory's compliance with Department of Transportation (DOT) regulations and the American Society of Mechanical Engineers (ASME) pressure vessel code

In addition to the above recommendations, the study also concluded that additional characterization is not likely to result in lower bids by prospective vendors, and that direct sampling of many older tails cylinders may be impractical.

The peer review team found that the ORNL general characterization strategy was statistically acceptable with qualification. It is surmised that with appropriate use of the historical information provided in the study, confidence parameters can be significantly upgraded, possibly with a reduction in cost. Furthermore, the bounding concentrations given for neptunium $(\mathrm{Np})$, plutonium $(\mathrm{Pu})$, and technetium in Table 3 [Hightower 2000], while extremely conservative for $\mathrm{DUF}_{6}$ that leaves the tails cylinders and is processed at a conversion plant, may or may not be bounding for some cylinders' contents in storage, depending upon the situation.

It is the peer review team's conclusion that the presence of americium 243 $\left({ }^{243} \mathrm{Am}\right)$ in the sampling results is an analytical artifact. Furthermore, the team concludes that while it is possible that americium $241\left({ }^{241} \mathrm{Am}\right)$ is present in the heels due to the decay of plutonium $241\left({ }^{241} \mathrm{Pu}\right)$, it is not likely.

The peer review team concluded that there might be limited value to conducting course of action (a) but was informed during this review that it is already in progress. The peer review team is in full agreement with courses of action (b) and (c) of the study. 
The peer review team concurs with the ORNL study's conclusion that there seems to be enough information for potential vendors to draw reasonable conclusions regarding their risk due to presence of TRUs and technetium in the tails cylinders. The peer review team also agrees with the ORNL study that sampling of many older tails cylinders may be impractical. There are regulatory concerns and availability issues that must be considered and resolved prior to any sampling of the older tails cylinders. Correspondingly, these concerns are applicable to any $\mathrm{DUF}_{6}$ cylinder transportation and emptying operations in a conversion campaign.

The following are recommendations made by the peer review team:

- A peer review concerning the suitability of the $\mathrm{DUF}_{6}$ cylinders for transportation and for emptying operations in a conversion campaign is necessary. The suitability of the cylinders for these operations probably presents a greater uncertainty for the RFP than does the TRU and technetium contamination of the cylinders.

- An analysis of the analytical data from cascade improvement projects should be conducted to determine the deposition characteristics of neptunium inside the cascades.

- If it is not possible and cost effective to identify the cylinders or groups of cylinders previously used to store reactor return feed, all cylinders should be treated as potentially containing some plutonium, and the wash from cleaning the cylinders should be treated as potentially TRU waste.

- Unless it can be proven that neptunium was deposited in the cascade equipment or remained in the heels, its presence in the conversion plant should be assumed, although at much lower concentration than suggested in the subject report.

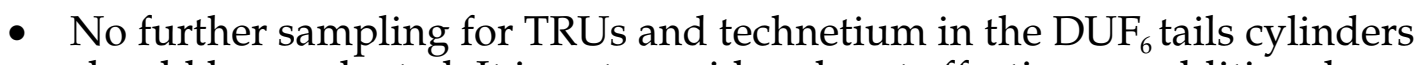
should be conducted. It is not considered cost effective, as additional characterization is not likely to result in lower bids by prospective vendors. 


\section{Introduction}

The Department of Energy (DOE) is preparing an RFP for the DUF 6 Conversion Services Project. Before the RFP can be issued, the type and amount of TRU and other radioactive material contamination in the $\mathrm{DUF}_{6}$ inventory must be determined. ORNL has developed the Hightower 2000 strategy for characterizing the extent of this contamination. The DOE tasked Argonne National Laboratory (ANL) and Lawrence Livermore National Laboratory (LLNL) to conduct a peer review of this strategy.

The Hightower 2000 study proposed a characterization strategy consisting of a general statistical sampling and analysis methodology, and six alternative characterization strategies. The general sampling methodology recommended a selection of twelve samples from six population groups of cylinders, which makes up the entire tails cylinder's inventory at each of the three gaseous diffusion plants. The six alternative characterization strategies are different combinations of varying information levels versus cost and scheduling constraints. The statistical sampling focused on providing an estimate of the probability that concentrations of contaminants in the tails material exceed a conservative bounding value. For the three principal isotopes of concern $\left({ }^{239} \mathrm{Pu}\right.$, ${ }^{237} \mathrm{~Np}$, and ${ }^{99} \mathrm{Tc}$ ), the Hightower 2000 study estimated the upper limit concentrations at $0.024,350$, and 100,000 parts per billion, respectively.

The Hightower 2000 study established confidence levels that minimize the sum of characterization costs, plus expected additional operating expenses that might be incurred as a result of contaminants in cylinders selected for processing outside these bounds. The Hightower 2000 study concluded that the only method of sampling analysis of cylinder contents which could provide all the information that vendors need, with the highest quality, is to retrieve vapor and liquid samples during the process of emptying a tails cylinder using autoclaves and cylinder washing for heels analysis.

The peer review identified the following as major conclusions presented in the ORNL study:

- Additional characterization is not likely to result in lower bids by prospective vendors

- Recent samples from three tails cylinders show detectable levels of americium

- Direct sampling of many older tails cylinders may be impractical

- A limited number of unused feed cylinders with reactor returns was identified that can be sampled

A complete list of the Hightower 2000 studies' recommendations can be found in Appendix A.

In addition to the above, the Hightower 2000 study proposed an alternative characterization strategy to their general strategy, considering cost and scheduling constraints. The Hightower 2000 study states this strategy would be sufficient to provide adequate information to support the RFP. This alternative characterization strategy consisted of the following recommendations: 
- Collect and analyze liquid samples from the two identified PGDP feed cylinders

- Subsequently, the DUF 6 should be fed into the cascades and an analysis conducted on the resulting heels by washing and analyzing the wash rinsate

- Characterize the cylinder coatings to determine the extent and levels of PCB contamination

- Verify the characterization of the cylinder inventory's compliance with DOT regulations and the ASME pressure vessel code

It is stipulated in the Hightower 2000 study that the measurements on these two cylinders would provide:

- Values of maximum likely concentrations of TRUs (including americium) in the $\mathrm{DUF}_{6}$ to be expected in tails cylinders

- The extent of transfer of TRUs expected to occur from cylinders into the conversion plants

- Values of TRUs in feed cylinder heels under conditions similar to those expected in some tails cylinders

- Additional information for understanding the mechanism for introduction of americium into the cascades

This peer review provides both general and specific observations to the Hightower 2000 general characterization strategy and its recommendations. These observations were made in the areas of TRU chemistry, statistical applications, and the uranium enrichment process. At the end of these observations are the peer review team's conclusions and recommendations.

A formal question and answer process was used to facilitate the peer review process. The peer review team composed a set of twelve questions to ORNL involving such topics as historical data, statistics, uranium chemistry, the presence of ${ }^{243} \mathrm{Am}$, and sampling techniques. Appendix B contains the peer review team's set of questions and ORNL's responses. Of particular interest to the peer review team was information concerning the history of tails cylinder number 137128. This was provided by ORNL and is located in Appendix C. Finally, attached to the end of this report are each reviewer's qualifications.

\section{Purpose}

The purpose of this peer review is to examine and prepare an evaluation of the data, assumptions, and process contained in the Hightower 2000 strategy. The review will determine if implementation of the strategy provides a realistic bounding of the extent of potential contamination in the DUF 6 cylinders, which is information necessary to issue the RFP. 


\section{General Observations}

The peer review evaluated the Hightower 2000 study in three main areas: TRU chemistry/radioactivity, statistical approach, and the uranium enrichment process. The observations presented in this section are portioned accordingly.

\subsection{Transuranic Chemistry/Radioactivity}

Based on the chemistry of the materials involved, the only TRU element that has a reasonable chance of making it to the tails cylinders through the cascades is neptunium. There are some potential reactions with some unknowns that could favor deposition and capture of neptunium in the cascades. The available analytical data seem to indicate that most of the neptunium may be plated out on the internal surfaces of the cascades. See Figure 3 [Parks 1999], pages 15-17 of [Smith 1984], and [Bailey 1988]. However, based on the chemistry of neptunium hexafluoride $\left(\mathrm{NpF}_{6}\right)$, its presence in the $\mathrm{DUF}_{6}$ cylinders cannot be ruled out. The presence of americium in tails cylinders is highly unlikely, and reported detection appears to be an analytical artifact, certainly for ${ }^{243} \mathrm{Am}$. While the plutonium contamination in feed may be sufficient to generate ${ }^{241} \mathrm{Am}$ in feed cylinders heels and washes, no such mechanism is likely for tails cylinders unless their prior use as feed cylinders is evident.

It is unlikely that there will be appreciable quantities of technetium in the tails cylinders, except some technetium can be expected in the heels and washes of those cylinders with prior history of use as reactor return feed cylinders.

The presence of other fission products in tails cylinders is not likely, even though there are volatile fluorides of fission products.

\subsubsection{Observations: Americium Contamination in Tails Cylinders}

The lower fluorides of americium are non volatile. If americium hexafluoride $\left(\mathrm{AmF}_{6}\right)$ was made (or existed), it can be assumed to be volatile but less so than the uranium, neptunium, or plutonium hexafluorides. It is highly unlikely that the conversion of uranium trioxide $\left(\mathrm{UO}_{3}\right)$ to $\mathrm{UF}_{6}$ will also produce $\mathrm{AmF}_{6}$. Further, it is expected that the $\mathrm{AmF}_{6}$ is quite unstable, and transfer to the feed cylinders is highly questionable. Moreover, retention in the feed cylinder as a volatile component, movement in the cascades, and return to the tails cylinders is so unlikely as to be effectively not possible. Therefore, even if there was americium in the $\mathrm{UO}_{3}$ from the separation plants, the likelihood that it transferred to the tails cylinder via a volatile fluoride is nil.

Since americium is likely to have ended up in the ash of the fluorinators, and no effective path to tails cylinder heels is evident; the only other source for americium is the ${ }^{241} \mathrm{Am}$ from the decay of ${ }^{241} \mathrm{Pu}$. What is not explained is the presence of ${ }^{243} \mathrm{Am}$, which is formed (only) from ${ }^{243} \mathrm{Pu}$ or berkelium $247\left({ }^{247} \mathrm{Bk}\right)$, neither of which seem feasible from weapons plutonium production. Berkelium does not form volatile fluorides.

In conclusion, it is possible that traces of ${ }^{241} \mathrm{Am}$ are present (have accumulated) in the heels of cylinders (not tails use only) in which plutonium-bearing $\mathrm{UF}_{6}$ was stored. The source would be decay of ${ }^{241} \mathrm{Pu}$. However, it is not reasonable that 
${ }^{243} \mathrm{Am}$ was derived from the reactor returns, and it is more likely that its "presence" is an analytical artifact.

\subsubsection{Observations: Plutonium Contamination in Tails Cylinders}

The assumption that approximately 0.1 grams of plutonium was actually fed into the cascades may be a satisfactory estimate, but the logic from [Smith 1984] seems to ignore the significant chemistry difference between neptunium and plutonium fluorides. The alpha activity ratio, adjusted for specific activities, roughly gives the answer in the reference. This estimate is high, and the quantity actually injected into the cascade at the feed point may be lower; however, the actual value may be of little importance. The chemistry of plutonium hexafluoride $\left(\mathrm{PuF}_{6}\right)$ is such that inside the cascade with the huge surface area, metallic materials, and the air in-leakage cited in some of the references, survival of a volatile form of plutonium seems extremely unlikely. Therefore, the expected transport of plutonium inside the cascade is highly likely to be limited to the feed point and a very small "distance" beyond it.

Since it is likely that plutonium was included in the $\mathrm{UO}_{3}$ from reactor returns, cylinders of $\mathrm{UF}_{6}$ used to hold the product from the tower reactors ${ }^{1}$ and that served as feed cylinders can be expected to contain in their heels finely divided plutonium in the form of plutonium tetrafluoride $\left(\mathrm{PuF}_{4}\right)$. All such cylinders should be treated as potentially containing some plutonium. Similarly, the wash obtained from cleaning such cylinders should be treated as potential TRU waste.

The $\mathrm{PuF}_{6}$ can be formed in the tower reactors although unless the geometry is well known, secondary reactions, and hence conversion efficiency of plutonium dioxide $\left(\mathrm{PuO}_{2}\right)$ to $\mathrm{PuF}_{6}$, is difficult to estimate. The $\mathrm{PuF}_{6}$ in equilibrium with fluorine and $\mathrm{PuF}_{4}$ (which is substantially insoluble, [five parts per billion] in $\mathrm{UF}_{6}$ ) in a feed cylinder should be very small. The fluorine pressure in feed or product cylinders is assumed to be nil. If, for example, a fluorine pressure in a cylinder is $1 \mathrm{E}-5$ atmospheres, the equilibrium $\mathrm{PuF}_{6}$ content approaches one part per billion, assuming no opportunities to react chemically or to remove the fluorine by chemical reaction. If there is an oxygenated uranium compound present, uranyl fluoride $\left(\mathrm{UO}_{2} \mathrm{~F}_{2}\right)$ for example, $\mathrm{PuF}_{6}$ will react rapidly. Thermal and alpha decomposition will also reduce the $\mathrm{PuF}_{6}$ content. In short, even if $\mathrm{PuF}_{6}$ were introduced into a feed cylinder, retention in that cylinder as $\mathrm{PuF}_{6}$ is highly questionable.

The plutonium content in "clean" tails cylinders can be expected to be below detection, as was found by [Taylor 2000]. It is possible in principle, but extremely unlikely, that finely divided plutonium fluoride transfers as dust through the cascade from the feed point to the tails withdrawal point. This was confirmed in [Taylor 2000]. However, this should not be anywhere near a measurable source of plutonium in the tails cylinders. Since cascade operations are on the gas phase of $\mathrm{UF}_{6}$, solubility is not an issue. What remains as needed information, therefore, is the use history of the cylinders.

\footnotetext{
${ }^{1}$ They were also known as "flame reactors" since the reaction rate was determined by how fast fluorine and the solid could be fed into the Monel tube. The reactions were often run at bright red heat of the tube and clearly represented a flame structure to the reaction zone.
} 
The plutonium content in cylinders used at any time to hold reactor return feed, such as material that came directly from tower reactors, can contain plutonium. Therefore, if tails cylinders have or are suspected to have had a history of service as reactor return feed cylinders, heels and wash could contain detectable quantities of plutonium.

In conclusion, the chemistry of $\mathrm{PuF}_{6}$ is such that a very modest fraction of the plutonium in the $\mathrm{UO}_{3}$ fed to the fluorinators would reach the feed cylinders, and a very small fraction of that would enter the cascades at the feed point. Transport of $\mathrm{PuF}_{6}$ through the cascades is highly unlikely, and hence, arrival of $\mathrm{PuF}_{6}$ at the tails withdrawal section should be so low as to be below detection. The presence of plutonium in tails cylinders when the source is cascade tails should be nil. It is possible that very finely divided plutonium fluoride solids could move through the cascade, but it is not credible. Contamination of plutonium in tails cylinders could reasonably only come from prior use of the cylinders to hold reactor return feed.

\subsubsection{Observations: Neptunium Contamination in Tails Cylinders}

Neptunium was not recovered as a useable product from much of the solvent extraction operations at Hanford. The presence of neptunium in the $\mathrm{UO}_{3}$ reactor returns is, therefore, not surprising. The tower reactors will easily convert neptunium dioxide $\left(\mathrm{NpO}_{2}\right)$ to neptunium hexafluoride $\left(\mathrm{NpF}_{6}\right)$, at high temperatures that should be quite efficient. If there were a purification step between generation of $\mathrm{UF}_{6}$ in the tower reactors and its feeding to the cascades, most of the neptunium would be removed from the $\mathrm{UF}_{6}$. However, based on the responses provided by ORNL to the questions and answers regarding the review of the subject report, it seems that such a step was not uniformly provided at the gaseous diffusion plant (GDP) sites. Therefore, the assumption in [Smith 1984] that 25percent (4.6 kilograms) of the neptunium received (18.4 kilograms) in the $\mathrm{UO}_{3}$ will enter the cascade, could be low.

Due to reactions with cylinder walls and depending on the availability of moisture in the feed cylinders, it should be expected that some of the $\mathrm{NpF}_{6}$ would have converted to non-volatile compounds and remained in the heels when the $\mathrm{UF}_{6}$ was fed to the cascades. While $\mathrm{NpF}_{6}$ is more reactive than $\mathrm{UF}_{6}$, it is stable enough to move in the cascade. Reaction with air will result in a nonvolatile oxyfluoride and deposition inside the cascade from this source, and, to a lesser extent, reaction with materials of construction can be expected. It may be that the neptunium-bearing dust loading of the barrier is attributable to a reaction with uranium compounds.

The relative stability of $\mathrm{NpF}_{6}$ makes it likely that it can move substantially through the cascades. As in the case of uranium, air in-leakage will deposit nonvolatile neptunium inside the cascade. Cascade equipment withdrawn from the cascade will show such deposits. It is not unreasonable to assume that some $\mathrm{NpF}_{6}$ will arrive at the tails section and hence be drawn into the tails cylinders, even though [Taylor 2000] and [Smith 1984] indicate none was detected. It is likely, however, that much less than 100 percent of the neptunium feed will arrive at the tails section. Further, if, as is likely, the tails cylinders will contain solid uranium compounds, it can be expected that significant neptunium reaction 
can take place over time and a deposit of neptunium, neptunyl fluoride $\left(\mathrm{NpO}_{2} \mathrm{~F}_{2}\right)$, for example, in the cylinder heels can be expected. However, [Taylor 2000] indicates none was detected. The relative stability of $\mathrm{NpF}_{6}$ will indicate, however, that considerable fraction of the neptunium in the tails will be in the form of $\mathrm{UF}_{6}$, with which $\mathrm{NpF}_{6}$ is expected to produce a continuous solid solution. Again, none was detected.

In conclusion, neptunium present in the reactor returns $\mathrm{UO}_{3}$ will convert readily to $\mathrm{NpF}_{6}$, and a high fraction will enter the cascade. Movement in the cascade should be in concert with $\mathrm{UF}_{6}$, but reaction with water and air might be more extensive than for $\mathrm{UF}_{6}$. Deposits of neptunium hydrolysis compounds (neptunyl fluoride for example) on barrier and equipment are likely. Withdrawal of some $\mathrm{NpF}_{6}$ into tails cylinders should be evident. However, available data indicate that neptunium concentration in the tails cylinders may be near or below the detection limit of five parts per billion.

\subsubsection{Observations: Technetium Contamination in Tails Cylinders}

The presence of relatively large amounts of technetium in the reactor returns $\mathrm{UO}_{3}$ will result in corresponding quantities of technetium trioxide fluoride $\left(\mathrm{TcO}_{3} \mathrm{~F}\right)$ or technetium hexafluoride $\left(\mathrm{TcF}_{6}\right)$ being formed and injected into the cascades at the feed point.

It is expected that much of the technetium would end up in the purge cascade above the $\mathrm{UF}_{6}$ withdrawal section. However, [Parks 1999] indicates (inconsistently) that technetium ended up in the top of the Oak Ridge GDP cascade where the product is withdrawn. There is no reason why significant or even measurable amounts of technetium should end up in the tails cascade section and, hence, the tails cylinders. This seems to be borne out by lack of detection of technetium in tails DUF $_{6}$ or heel washes [Smith 1984] but reported as detected in [Taylor 2000]. The latter specified clean cylinders without prior use to hold feed $\mathrm{UF}_{6}$.

In conclusion, the behavior of technetium seems to follow the obvious chemistry. Highly efficient conversion to a volatile fluoride/oxyfluoride is reasonable. Lack of evidence for technetium in the tails cylinders is also in accord with expectations, but data are confounding. Transport of solid, powdered technetium deposits through barrier and equipment to the tails section seems not feasible. Some technetium in the tails cylinders could be expected if the cylinders had been used for reactor return feed.

\subsubsection{Observations: Fission Product Contamination in Tails Cylinders}

While nuclides such as zirconium 95, ruthenium 106, cesium 137, and cerium 144 were detected in fluorinator ash, there is little reason to expect to find any of them in the tails cylinders, unless these cylinders have been used for reactor return feed to the cascades. Of the isotopes noted above, only ruthenium forms volatile fluorides. These (ruthenium tetrafluoride oxide $\left(\mathrm{RuOF}_{4}\right)$ m.p. $115^{\circ} \mathrm{C}$, b.p. $184^{\circ} \mathrm{C}$; ruthenium pentafluoride $\left(\mathrm{RuF}_{5}\right)$ m.p. $90 \mathrm{C}$, b.p. $270^{\circ} \mathrm{C}$; ruthenium hexafluoride $\left(\mathrm{RuF}_{6}\right)$ m.p. $54^{\circ} \mathrm{C}$ no b.p. data) are likely to form and partition between the ash and the $\mathrm{UF}_{6}$. Since ruthenium is hard to remove from reprocessing products and is likely to be there as the dioxide, ruthenium 
tetraoxide $\left(\mathrm{RuO}_{4}\right)$ is volatile and somewhat unstable; its presence is not out of the question. However, the isotope has a one-year half-life and at this point should be substantially decayed.

There are other fission products that have volatile fluorides. These include niobium pentafluoride $\left(\mathrm{NbF}_{5}\right)$, tellurium hexafluoride $\left(\mathrm{TeF}_{6}\right)$, molybdenum hexafluoride $\left(\mathrm{MoF}_{6}\right)$, molybdenum tetrafluoride oxide $\left(\mathrm{MoOF}_{4}\right)$, and antimony pentafluoride $\left(\mathrm{SbF}_{5}\right)$, in addition to those already mentioned. None of these seem to represent a contamination hazard, but no information could be found about their chemical presence and their influencing the use of the depleted uranium product. Some of the fission products may be detectable by non-destructive assay methods since they have characteristic hard gammas. However, the background with uranium may be too high for sensitive detection.

\subsection{Statistical Approach Observations}

The statistical review focuses on the sampling plan outlined in the Hightower 2000 study. In the study, the key guiding principles for the selection of the general statistical sampling method and analysis are:

"To sample from the worst cases and use them as a basis for conservative conclusions about the population of cylinders in general..."

"The extent of sampling should balance sampling costs with the payback for having sample analysis results."

While the peer review agrees with these guiding principles, the statistical observations are based upon them.

While the balancing act referred to can be implemented by a deft use of operational analysis techniques, the choice of the "worst cases" referred to may, in general, be difficult, depending to a large extent on the intimate process knowledge of the practitioner. ORNL personnel, however, have years of experience in such characterizations, and it is expected that they will adequately ensure the correctness of such "worst case" characterizations.

The cost/benefit analysis in Section 7 [Hightower 2000] leading to the "optimal" triple $(X, Y, n)$ for a range of cost parameters is both innovative and convincing, assuming that the optimization has been carried using exactly two of the triple $(\mathrm{X}, \mathrm{Y}, \mathrm{n})$ as independent variables. The analysis leads to a recommendation of the confidence parameters $X=85 \%$ and $Y=15 \%$, using a sample size of $n=12$ per subpopulation. The review team recommends that, to make the cost factor more transparent, it may be worthwhile to add a table showing the total cost for a range of cost parameter values using the optimal choice for the triple.

It should be noted that the rather moderate sample size of 12 recommended in the optimal plan comes at the expense of significantly degraded confidence parameters, namely $(85,15)$, with the associated consequences.

The strategy of sharpening of the optimal sample size using the sigmoidal curve argument mentioned in Appendix C [Hightower 2000] is inoperative because of technical reasons. Among them are: 
- $\mathrm{Z}$ being the maximum concentration in a sample is random. Therefore, with regard to the survivor function $\mathrm{P}(\mathrm{C}>\mathrm{Z})$, the fixed parameter in a distribution is not being considered.

- The sigmoidal and other assumptions of the plot of the survivor function are too restrictive. They are not supported by empirical data. Even the cited parametric concentration distributions do not, in general, satisfy these conditions. (Take for example, a Weibull distribution with the shape parameter less than or equal to one.)

Thus, the payback with the sample analysis results cannot be improved with a cost reduction using this strategy. To this end, the following comment may be noted:

Very little explicit use has been made of the modest amount of prior information available in Tables 1-3 and Table C1 [Hightower 2000]. There are additional prior information sources alluded to in the proposal. It is the review team's contention that with appropriate use of the prior information, including those in the tables indicated, confidence parameters can be significantly upgraded and done so with a possible reduction in cost.

\subsection{Uranium Enrichment Process}

The report by R.F. Smith [Smith 1984] entitled "Historical Impact of Reactor Tails on Paducah Cascade" is one of the key references used in generating the subject report. On pages 15-17 of that reference, there is a discussion on sampling and analysis of two tails cylinders at the PGDP that were filled during June 1973, when more than 96 percent of the feed to the cascades was from reactor returns. According to the reference, reactor returns constituted approximately 65 percent of the feed to the cascade in fiscal year 1973 and about 27 percent for the preceding year.

The analytical data that was reported in [Smith 1984] indicated that the concentrations of technetium, ${ }^{237} \mathrm{~Np}$, and ${ }^{239} \mathrm{Pu}$ in those two tails cylinders were found to be less than 1 part per billion for ${ }^{99} \mathrm{Tc}$ and ${ }^{237} \mathrm{~Np}$, and less than 0.01 part per billion for ${ }^{239} \mathrm{Pu}$. The conditions under which these two cylinders were filled can probably be considered as reasonably conservative, and the measurements considered to be a good indication of the difficulty for these contaminants making it to the depleted stream through the cascades. This important piece of information was overlooked (see the first question in Appendix B) by the authors of the subject report. The authors indicated that they would discuss these data in the final report.

If any of the depleted $\mathrm{UF}_{6}$ cylinders in current use were previously used to store reactor return feed material and were not cleaned before being filled with cascade tails, then those cylinders are very likely to contain TRUs and technetium. However, these contaminants are expected to be non-volatile and are expected to stay in the heels when the depleted $\mathrm{UF}_{6}$ cylinders are emptied for conversion in the gas phase. There may be small quantities of TRUs that may be entrained as particulate matter, and it may be necessary to install filters to trap these entrained particulates when the cylinders are emptied. 


\section{Specific Observations}

This section addresses specific observations to the Hightower 2000 study. In front of each observation, a reference to the location in the subject report or its references is provided.

\section{In regards to Pages 5-7 (Tables 1-3) and Appendix C [Hightower 2000]:}

A significant amount of quantitative information is available based on prior analysis of cylinder data as evidenced in Table 1-3 and Table C1. Others have been alluded to in the proposal. Not using this information explicitly when possible restricts us to extremely conservative conclusion, leading to cruder confidence levels and larger sample requirements. These, in turn, lead to higher costs. The appropriate tool to use the prior information is provided in Bayesian statistics. Here, the prior information regarding the parameter to be estimated is captured in an appropriate prior distribution, and when new observations come in, they are used to update the prior distribution to one called the posterior distribution, from which confidence intervals for the parameters under investigation can be computed. As an example, suppose it is estimated that $\pi=$ Prob (a contaminant concentration exceeds the detection level). Based on the data in Table $\mathrm{C} 1$, an appropriate prior for $\pi$ may be chosen as beta $(0.993,151)$. Suppose now, in a sample of size 12, no cylinder is detected with a contaminant concentration exceeding the detection level. The updated distribution for $\pi$ is a beta $(0.993,163)$. If instead, one cylinder in the sample had a contaminant concentration exceeding the detection level, then the corresponding posterior distribution for $\pi$ will be beta $(1.993,162)$. The prior and the two posteriors are shown in the figure below.

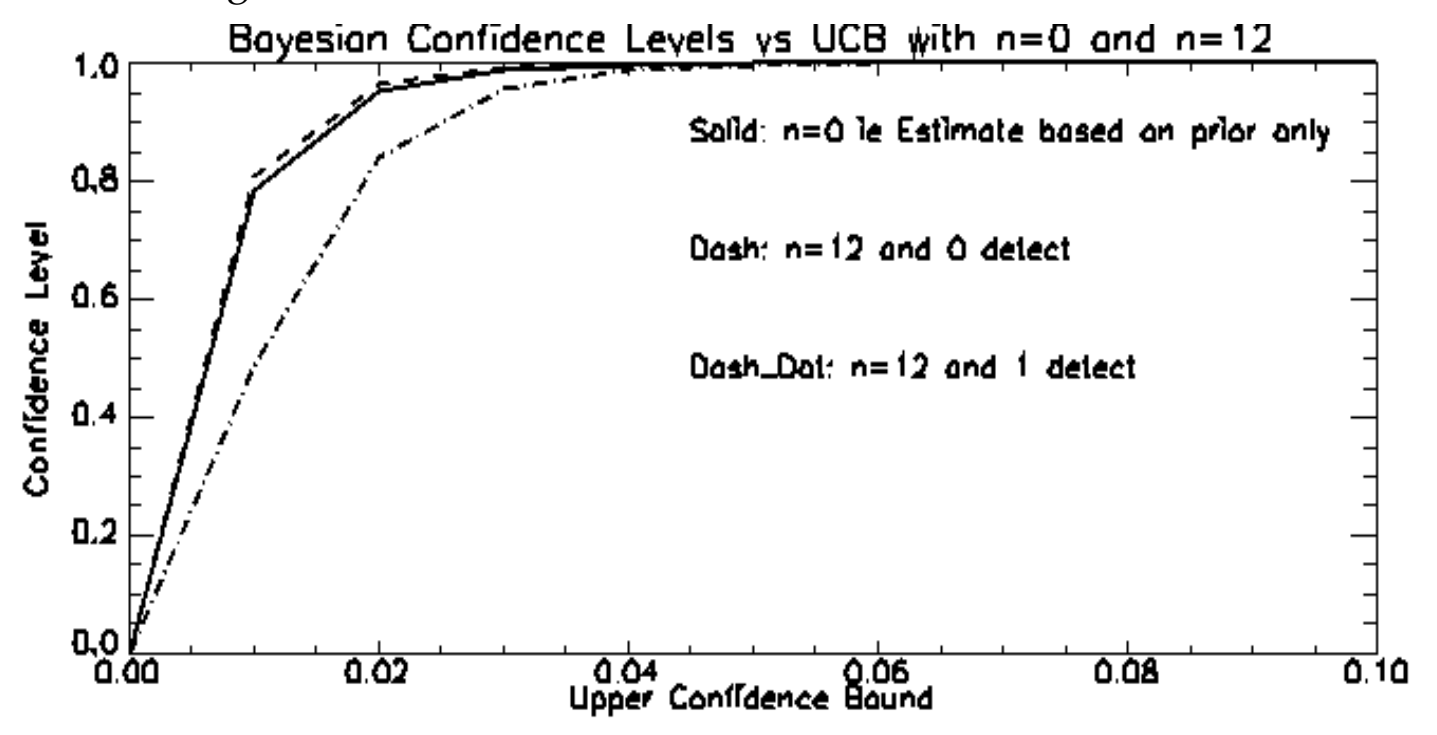

Notice that the confidence parameters are significantly improved with the same sample size as recommended in the proposal. Some confidence parameter pairs that can be read approximately from the figure are $(85,1)$ and $(99,2)$ for no "detect" and $(85,2)$ and $(99,3)$ for one "detect." 
Among other advantages offered in the Bayesian approach, for this particular problem, is the simultaneously selection, in an objective manner, the "worst case" and an estimate of the parameter $\pi$ using a finite number of samples.

In regards to Page 7, Table 3 and text below the table, and Appendix A, pp. 35-38 [Hightower 2000]:

The bounding concentrations given for neptunium, plutonium, and technetium may or may not be bounding. The assumptions made in Appendix A, on page 37 , are contrary to the facts. All of the TRU nuclides and technetium do not actually get into the cascades, and those that do, do not all make it to the tails. In fact, very little of these contaminants that exist in the feed cylinders actually make it to the tails through the cascades. The major source of transuranics and technetium in the tails cylinders is expected to be the heels that were left in the cylinders, after being used to store feed material. If a feed cylinder was used more than once to carry feed to the cascades before being retired to be a tails cylinder, and if it was not washed between feedings, then there would be an accumulation of non-volatile contaminants in the cylinder, and the values given in the report may not be bounding.

Another implicit and incorrect assumption made in the subject report is that the TRUs and technetium would be uniformly distributed in the tails cylinders. In fact, these contaminants, because of their relatively high melting points, would not liquefy when the cylinders were filled with depleted $\mathrm{UF}_{6}$. Some of them $\left(\mathrm{PuF}_{4}\right.$ for example) are insoluble in $\mathrm{UF}_{6}$ and would not dissolve. If they were stirred up due to incoming liquid depleted $\mathrm{UF}_{6}$, they would tend to settle back towards the bottom during the cool-off period of the filled cylinder. This is due to the density difference between the solid heel products and the low viscosity of liquid $\mathrm{UF}_{6}$. As a result, the concentrations of contaminants would tend to be greater in the bottom half of the cylinder than on top.

In regards to Pages 12-14, Cylinder Handling Characteristics [Hightower 2000]:

Uncertainties discussed with regards to handling characteristics of the cylinders are acknowledged.

\section{In regards to Page 26, and Table 7 on Page 29 [Hightower 2000]:}

The cost for Alternative 3 is given as $\$ 540,000$ in the text but it is stated in the Table as $\$ 660,000$. Similarly, the cost for Alternative 4 is given as $\$ 660,000$ on page 26 , but the table lists it as $\$ 780,000$.

\section{In regards to Section 5 and Section 7 [Hightower 2000]:}

No part of the statistical analysis in Appendix $C$ has been used in the statistical sampling plan described in Section 5, and optimum number of samples, and confidence levels selection in Section 7.

\section{In regards to Paducah Lab Reports [Taylor 2000] and ORNL's interpretation of} the results:

Paducah Lab reports are difficult to understand and interpret. It appears that the interpretation provided by ORNL for americium isotopes may be incorrect, and the presence of these isotopes in the samples identified may be false positives. 
On the Cylinder Wash Rinsate results (Samples WC9-171 and WC9-171D), americium 243 counting errors are about twice as large as the reported results. This type of reporting generally indicates problems with resolution and noise. The gamma spectrometers are programmed to assign peaks at certain energies to certain radionuclides. Am-243, if present, emits gammas with $74.67 \mathrm{KeV}$ energy (branching ratio $=0.66$ ). Normally, a peak at $75 \mathrm{KeV}$ is assigned to Am-243. There are possibly six radionuclides present in the wash rinsate sample that had gammas very near the $75 \mathrm{KeV}$ peak. These radionuclides, their gamma branching ratios, and gamma energies are listed below.

Thorium 234 and protactinium $234 \mathrm{~m}$ are short-lived daughters of uranium 238, and were probably in secular equilibrium with U-238 in the sample. Lead 214 is also a member of the U-238 decay chain, although it is farther removed from U-238, and its concentration is expected to be less than others are. The spectrometer very likely had difficulty with resolving the gammas, and the software library mistakenly identified Am-243 as the corresponding radionuclide. The relatively large counting errors reported are an indication that the observed peak was not well defined.

\begin{tabular}{|l|l|l|}
\hline \multicolumn{1}{|c|}{ Radionuclide } & \multicolumn{1}{|c|}{ Branching Ratio } & Gamma Energy $\mathbf{( K e V )}$ \\
\hline Th-234 & $1.1 \mathrm{E}-04$ & 73.92 \\
\hline Th-234 & $3.9 \mathrm{E}-04$ & 74.00 \\
\hline $\mathrm{Pa}-234 \mathrm{~m}$ & $1.1 \mathrm{E}-04$ & 73.92 \\
\hline $\mathrm{U}-235$ & $6 \mathrm{E}-04$ & 74.96 \\
\hline $\mathrm{Pb}-214$ & $6.3 \mathrm{E}-02$ & 74.81 \\
\hline
\end{tabular}

Results reported for Am-241 are somewhat mixed. Both liquid samples and one of the duplicate samples from the wash rinsate suffer from the same type of uncertainty and possible interference with other radionuclides. Non-matching duplicates may be an indication of non-homogeneous sample or an analytical measurement problem. One possible source of Am-241 would be beta decay from $\mathrm{Pu}-241$, although this seems remote considering there was little evidence of plutonium isotopes.

The meaning of the letters under columns "Result Qual" and "Foot Notes" is not given. Do "U" and "A" stand for Unacceptable and Acceptable, respectively? If so, the lab reports might be suggesting that some of the americium results should not be reported as real.

Is the "Reporting Limit" on Paducah Lab reports the same as the "Minimum Detectable Activity (MDA)" on Portsmouth reports? On liquid sample reports, the reported result for Am-241 for both cylinders and Am-243 result for Cylinder 135117 is less than the "Reporting Limit." It is not meaningful to report such results as absolute values. 


\section{Conclusions}

The peer review team finds that the general characterization strategy presented in the Hightower 2000 study possesses statistical merit. From an operational perspective, the cost/benefit analysis presented in the proposal is convincing. The indicated sampling plan meets a minimal set of confidence objectives from a statistical perspective as well, although significant improvements in terms of improved confidence parameters and/or reduced cost are possible. If additional sampling was conducted, the Hightower study's recommendation that the vapor and liquid sampling using the autoclave and cylinder washing is the best method to obtain the characteristics of both the gaseous and the heel material is strongly supported.

The bounding concentrations given for neptunium, plutonium, and technetium in Table 3 [Hightower 2000] may or may not be bounding depending upon the situation. The assumptions made in Appendix A [Hightower 2000] are contrary to the facts. Very little of the TRU nuclides and technetium contaminants get into the cascades or make it to the tails. Therefore, the study's bounding concentrations are very conservative when applied to the cylinders with no prior history as feed cylinders. The major source of TRUs and technetium in the tails cylinders is expected to be the heels left in the cylinders after being used to store feed material. If a feed cylinder were used more than once to carry feed and not washed in between, then non-volatile contaminants would accumulate and tend to concentrate at the bottom. As a result, the uniform concentrations given in the study would probably not bound the concentrations encountered in the bottom portion of such cylinders.

It is important to recognize that the conclusions and recommendations outlined in the Hightower 2000 study do not reflect their general characterization strategy. As an alternative, the study recommends as its characterization strategy to:

- Collect and analyze liquid samples from the two identified PGDP feed cylinders, feed the $\mathrm{UF}_{6}$ into the cascades, and conduct an analysis on the resulting heels by washing and analyzing the wash rinsate

- Characterize the cylinder coatings to determine the extent and levels of PCB contamination

- Verify the characterization of the cylinder inventory's compliance with DOT regulations and the ASME pressure vessel code

The peer review team was informed during this task that the first recommendation is already in progress. While additional sampling can supply more information, the value of this recommendation is in dispute. The study's supposition that the sampling of these two feed cylinders would provide likely measurement values for maximum TRU concentrations in the tails cylinders, transfer concentrations of TRUs from cylinders into the conversion plants, and TRUs in feed cylinder heels is not defensible from a statistical perspective. The sampling of one or two cylinders is not adequate to provide the necessary statistical justification of the statements expressed unless process knowledge of a quantitative nature is incorporated in the decision. 
However, if the history of the two feed cylinders can be correlated to a specified population group, the measured values could be a good indication of the TRUs in the heels of some tails cylinders. In particular, the measured concentrations of contaminants in the heels of the two feed cylinders would be indicative of the expected concentrations of the same contaminants in the heels of those depleted $\mathrm{UF}_{6}$ cylinders previously used as feed cylinders. The peer review team is in full agreement with the second and third recommendations of the study.

In addition to the above recommendations, the study proffers that:

- Additional characterization is not likely to result in lower bids by prospective vendors

- Recent samples from three tails cylinders show detectable levels of americium

- Direct sampling of many older tails cylinders may be impractical

The peer review team concurs with the Hightower 2000 study's first conclusion that there seems to be enough information for potential vendors to draw reasonable conclusions regarding their risk due to presence of TRUs and technetium in the tails cylinders. It is not clear that sampling of additional cylinders can provide a positive benefit/cost ratio. This statement is made with the postulation that the existing process knowledge introduced in the study is representative of the three GDP's entire cylinder population.

As long as the cylinders are intact, the incremental risk due to presence of TRU and technetium contaminants in the cylinders should be negligibly small. Should a cylinder be breached and spill some portion of its contents to the environment, it is likely that these contaminants would contribute to the radiation dose of the receptors. However, in a situation like this, the main hazard would be from chemical effects resulting from exposure to reaction products of depleted $\mathrm{UF}_{6}$ and moisture in the air (mainly $\mathrm{HF}$ and $\mathrm{UO}_{2} \mathrm{~F}_{2}$ ). The contribution of the contaminants to the chemical effects would be negligibly small. Therefore, sampling of the cylinders for the purposes of the cylinder management program would not be necessary.

Regarding the second conclusion, it is possible that traces of ${ }^{241} \mathrm{Am}$ are present (have accumulated) in the heels of cylinders (not tails use only) in which plutonium-bearing $\mathrm{UF}_{6}$ was stored. The source would be decay of ${ }^{241} \mathrm{Pu}$. However, it is not reasonable that ${ }^{243} \mathrm{Am}$ was derived from the reactor returns, and it is more likely that its "presence" is an analytical artifact.

The peer review team concurs with the Hightower 2000 study's third conclusion. There are regulatory concerns and availability issues that must be considered and resolved prior to any sampling of the older tails cylinders. Correspondingly, these concerns are applicable to any $\mathrm{UF}_{6}$ cylinder transportation and emptying operations in a conversion campaign.

\section{Recommendations}

Unless the DOE is reasonably assured, based on the review done by the Defense Nuclear Facility Safety Board, it would serve the DOE well if the data and documentation concerning the suitability of the depleted $\mathrm{UF}_{6}$ cylinders for 
transportation and for emptying operations in a conversion campaign were subjected to a peer review by outside experts. The suitability of the cylinders for these operations probably presents a greater uncertainty for the RFP than does the TRU and technetium contamination of the cylinders. A review of Section 4.4 and Appendix B of the subject report, and a cursory review of an internal correspondence report referenced in Appendix B as B5 [S.J. Pawel to M.S. Taylor, "Cylinder Condition Acceptance Requirements for Multiple Cylinder Functions," ORNL/CST-SP-072998-01, July 29, 1998] seems to indicate that much of the documentation and rationale developed for this purpose is based on internal correspondence and unpublished reports. A peer review of the available data and timely identification of data needs and gaps, accompanied by a concerted effort to obtain the needed data, would reduce the chances for surprises and costly delays later in the conversion program.

The only plausible pathway for TRUs and technetium to end up in the depleted $\mathrm{UF}_{6}$ cylinders is by way of heels from prior use of the cylinders to store reactor return feed. One possible exception to this is neptunium, although the available data seem to indicate that the same conclusion should hold for this radionuclide. It is recommended that as much as possible of the analytical data from cascade improvement projects be analyzed to determine the deposition characteristics of neptunium inside cascades. The Environmental Health Office of Oversight's Mass Balance Study could also shed some light on this issue.

Because the contaminants in the heels are nonvolatile, they are expected to remain in the cylinders or become captured in filters when the cylinders are emptied at a conversion plant. For the purposes of the RFP, it should be assumed the heels from all cylinders suspected to have a history of use as reactor return feed cylinders, and any filters used in the feed stream to the conversion plant, will contain TRUs. Depending on the assays performed during conversion, the wastes from these cylinders and filters may or may not be TRU waste. If it is possible and cost effective to identify the cylinders or groups of cylinders previously used to store reactor return feed, those cylinders should be identified. If it is not possible or cost effective, then the peer review team recommends that all cylinders should be treated as potentially containing some plutonium, and wash from cleaning cylinders should be treated as potentially TRU waste.

The depleted $\mathrm{UF}_{6}$ processed at a conversion plant after it leaves the cylinders should be essentially free of TRUs and technetium. Again, one possible exception is neptunium. The concentrations of the contaminants in the gaseous depleted $\mathrm{UF}_{6}$ that leaves the tails cylinders are expected to be near or below the limits of detection. Therefore, it is recommended that the processed material be subjected to no special handling other than what would be required for pure depleted $\mathrm{UF}_{6}$.

It is recommended that no further sampling for TRUs and technetium in the DUF $_{6}$ tails cylinders be conducted. It is not considered cost effective, as additional characterization is not likely to result in lower bids by prospective vendors. 


\section{References}

Bailey, J.C. (1988), "Radionuclides in the Equipment of the Oak Ridge Gaseous Diffusion Plant," Presentation to DOE Oak Ridge Operations and DOE Headquarters at the ORGDP, March 10.

Hightower, J.R., et. al. (2000), "Strategy for Characterizing Transuranics and Tc Contamination in UF 6 Tails Cylinders," Draft, Oak Ridge National Laboratory, April 7.

Parks, J.W., et. al. (1999), "Recycled Uranium Processed at the Oak Ridge, Paducah, and Portsmouth Gaseous Diffusion Plants (Draft)," Interim report, September.

Smith, R.F. (1984), "Historical Impact of Reactor Tails on the Paducah Cascade," KY/L-1239, March.

Taylor, M.S. (2000), Personal communication to J.R. Hightower, January 21. 


\section{Appendix A: Hightower 2000 Study Conclusions}

- For bid preparation, vendors need information on the upper bounds of quantities of transuranic and fission product contamination that can be transferred from cylinders to the conversion plant and quantities of contamination that will remain in the cylinders as heels

- Because reported evidence indicates that levels of contamination in the $\mathrm{UF}_{6}$ are likely to be near or below levels of detection, the most important information needed is bounding information on the amount and contaminant content of heels that will already exist or will form in emptied cylinders

- The cylinders that are most likely to have the highest levels of the contaminants of concern and thus have the highest priority for characterization are an estimated 9,100 cylinders generated at the PDGP in 1957, 1965, 1969-1970, and 1972-73

- New results from liquid $\mathrm{UF}_{6}$ samples from PDGP cylinders filled during a period when there were no reactor returns being fed indicate the somewhat surprising presence of americium isotopes at levels that could be of concern

- The best known approach to obtain the needed information is by direct measurement during the process of emptying a cylinder (in the same manner as would be employed in the conversion plant) and from the analysis of heels material left behind at a cost per cylinder of about $\$ 20,000$

- The only readily available equipment for making the needed measurements belongs to USEC, and the schedule for its use is dictated by USEC needs and constraints

- Tc-99 should not pose a significant exposure hazard in a depleted uranium stream but could impact future beneficial use of the depleted uranium

- Transuranics can potentially concentrate in heels that remain in cylinders at levels high enough to require this material to be managed as TRU waste

- The cost impact for managing heels as TRU waste is relatively low, with expected incremental costs to be approximately $\$ 100-\$ 400$ per cylinder that contains elevated levels of transuranics

- Because of the high cost per cylinder for analysis and the low incremental cost impact of uncertainties in characterization results, statistical sampling and cost/benefit considerations show that only a small number of cylinders is economically justified to be selected for measurement

- Additional characterization is not likely to result in lower bids or affect the potential for change orders by providing better estimates of amounts of TRU wastes generated

- Additional measurements are desirable to help understand the significance of the new results that indicate a presence of americium 


\section{Appendix B: Initial Q\&A Regarding the Review of the "Strategy for Characterizing Transuranics and Tc Contamination in $\mathrm{UF}_{6}$ Tails Cylinders"}

The DOE has tasked a joint LLNL/ANL team to review [Hightower 2000]. The following questions have been prepared to facilitate this review process.

Question: The report by R.F. Smith entitled " Historical Impact of Reactor Tails on Paducah Cascade" is one of the key references used by ORNL in generating the subject report. On pages 15-17 of that reference, there is a discussion on sampling and analysis of two tails cylinders at PGDP that were filled during a month in 1973 when more than 96 percent of the feed to the cascades was from reactor returns. Despite the fact that this report is heavily relied on for data concerning the feed rates into the cascades, there is no mention of the sampling and analysis results from these two tails cylinders. This type of data would be most relevant to the problem at hand. Why was this data not used by the authors of the Hightower 2000 report? Is there a problem with the quality of the mentioned data?

Response: The discussion by R.F. Smith of the sampling of the two cylinders that were filled in 1973 was overlooked. We agree that these results are significant and should be discussed in section 3.1. A discussion summarizing the results will be added to section 3.1. This result strengthens the conclusions of the report.

Question: What is the history of the Cylinder No. 137128 sampled for heels (see Table 1 and Reference 2 in main report)? Is it possible that this cylinder was previously filled with reactor returns?

Response: The history of this cylinder has not yet been retrieved from records and compiled. Bechtel Jacobs Company, the DOE contractor responsible for the cylinder management program, would need to be authorized to compile a history of cylinder 137128 from archived paper records. We need to have this information to help develop an explanation of the analysis results.

Question: Are there any analytical data from cascade improvement projects that would show the degree to which impurities plated out on internal surfaces of the cascade equipment?

Response: Reference 1 from the characterization strategy refers to such information. We are continuing to look for additional existing information pertinent to this strategy. A recently-identified unpublished review of isotopic contamination found at the gaseous diffusion plants summarizes accessible contamination sampling data at K-25; this summary indicates the presence of ${ }^{241} \mathrm{Am}$ (but not ${ }^{243} \mathrm{Am}$ ) in the cascade equipment. In addition, telephone conversations with BNFL staff involved in the D\&D operations in K-33 report 
measured surface contamination of Tc and $\mathrm{Np}$ at levels near the detection limit. At these low levels these contaminants do not affect the D\&D operations.

Question: Was there a purification step included at any of the sites where the $\mathrm{UF}_{6}$ produced by the fluorination process was subjected to absorption/desorption reactions in $\mathrm{NaF}$ or in some other trap?

Response: A literature survey on removal of Tc, $\mathrm{Np}$, and $\mathrm{Pu}$ from $\mathrm{UF}_{6}$ can be found in ORNL/TM-5913, Laboratory and Pilot-Plant Studies on the Conversion of Uranyl Nitrate Hexahydrate to UF 6 by Fluidized-Bed Processes, by E. L. Youngblood, I. J. Urza, and G. I. Cathers, June 1977. This survey and other information indicate that a strong development program for trapping these constituents from $\mathrm{UF}_{6}$ streams was in place from about 1960 and considered trapping by $\mathrm{CoF}_{2}$, $\mathrm{MgF}_{2}, \mathrm{NaF}$, and other sorbents. Reference 6 of the characterization strategy is also a technical report on one of the development activities. We have no information as yet about what processes, if any, might have been actually utilized.

Question: Is it possible to identify which tails cylinders were previously used to store feed $\mathrm{UF}_{6}$ from recycling operations?

Response: It is possible to do so by analysis of the paper history for each cylinder to determine which cylinders started out as feed cylinders and whether they ended their service in tails storage without being washed first. This would be a labor-intensive time-consuming activity. It has been suggested that all such cylinders were 30-inch feed cylinders. This has not been confirmed; but if this were the case, the task would be limited to an examination of the records for only about 1700 cylinders rather that for all cylinders (approx. 50,000). The decision to do this will likely require prioritization among potential follow-on tasks.

Question: Because Am-243 is an uncommon isotope, the question of not only why it is there is a puzzle, but also it would be helpful to know if this radionuclide is present elsewhere in the process or even on the site.

Response: This information is not readily obtainable. A recently-identified unpublished review of isotopic contamination found at the gaseous diffusion plants identified ${ }^{241} \mathrm{Am}$ as a contaminant. None of the available information indicates that the presence of ${ }^{243} \mathrm{Am}$ was ever considered or sought. A question arises as to why the ${ }^{243} \mathrm{Am}$ was even on the list of nuclides to be measured in the PAD tails cylinders. This question will be referred to the Paducah analytical laboratory. Potential sources of ${ }^{241} \mathrm{Am}$ that were considered included old industrial smoke detectors, but it was concluded that the most likely source was ${ }^{241} \mathrm{Pu}$ present in reactor returns but not recognized or measured during the time that reactor returns were being processed. One other possible source of information is from on-going mass balance studies. 
Question: Again pertaining to Am-243, is it possible that while this was reported as a real value, it was instead an artifact from adding an isotopic dilution spike in the analytical procedure?

Response: This question will be referred to the Paducah analytical laboratory.

Question: The sampling technique of choice for heels analysis in the cylinders is a water wash. Because most fluorides are not readily dissolved in water, how much of the material is removed from the cylinder using this method? If the material is an oxide or other compound how much is dissolved or removed as a slurry? In other words, is the sample taken from the cylinder representative; is the sample complete, or is there a fractionated residue?

Response: The question enumerates recognized deficiencies with the technique for heels analysis. Without investing in additional facilities and capabilities, we are limited to a large extent to trying to extract useful information from procedures that were developed for other purposes in a production environment. There may be some improvements in future washings that can be accommodated, such as acidifying the wash solution to maximize solubility of constituents of interest.

Question: You have created six mutually exclusive populations of tail cylinders for statistical sampling purposes (starting pg. 17). There are three for PGDP: (1) pre-1973, high risk, processing reactor returns (RR), (2) pre-1973, all other years, and (3) 1973 and later, all years. There is one unique to ORGDP: (4) all years processing RR. There is one unique to PORTS: (5) all years processing RR. The last population is common to ORGDP and PORTS: (6) all other years for ORGDP and PORTS. For PGDP, it is understood that documentation and sample analysis is only available for post-1973 cylinders and your population choices are logical. The choice of high-risk populations (4) and (5) is also logical, assuming the distinction is made by the production plant doing the processing. Population (6), the low-risk years, combines two production plants in one population. Following this logic, should not (2) be combined with (6)? Alternately, following the logic in choosing (1), (2), and (3), should (6) be separated into two populations based on production plant? Please explain your logic in choosing the six populations as there appears to be possible inconsistencies.

Response: The better choice for population (6) would be to separate it into two populations based on production plant as is suggested. However, it is felt that this population is of such low priority for sampling and analysis that, for the present, discussing it as a single population is sufficient for outlining the general strategy for characterization.

Question: Choice of the null and alternate hypotheses: The Neyman-Pearson proposal for testing statistical hypothesis starts with a small positive number $(\alpha)$ such that probabilities of type I error greater than $\alpha$ is undesirable. This is so because lowering one of the error probabilities comes at the cost of raising the 
other and by convention, error of type I is considered a more "serious" error than an error of type II. In the section entitled "Theoretical Analysis" (pg. 47), the stated null and alternative hypotheses are those for a binomial parameter $\pi$ (actually it is a hypergeometric parameter, but since the distribution is well approximated by a binomial under the conditions of the problem, we can test equivalently for the binomial parameter $\pi$.)

$\mathrm{H}: \pi=\mathrm{p}$ vs. $\mathrm{K}: \pi>\mathrm{p}$

where $\pi=\mathrm{P}\left(\mathrm{C}>\mathrm{c}^{\prime}\right)$ for a suitable choice of $\mathrm{c}^{\prime}$.

Note that with $\mathrm{K}$ fixed as in above the resulting test will be the same if we replace $\mathrm{H}$ by $\pi<=$ p.

This implies that the more serious kind of error (error I) is accepting $\mathrm{K}$ when in fact $\mathrm{H}$ is true. It also implies that the probability of its happening is to be bounded above by a small positive number (the designated level of the test) while minimizing P (error II).

Based on the above, it is clear that either the description of the two types of error in the beginning of paragraph 2 of the section are switched, or the hypotheses $\mathrm{H}$ and $\mathrm{K}$ are switched.

Response: Appendix $\mathrm{C}$ is an exposition of the statistical properties of the tests. The relative importance of Type I and Type II error is neither implicitly nor explicitly assumed. The "I" and "II" in the sentence at the beginning of paragraph 2 will be switched to make the sentence consistent with the discussion before and after it.

Question: Which of the above switches is the case? The computations later seem to indicate that it is the description of the two types of error that is erroneous. Nevertheless, it is important to avoid the more serious error from happening.

Response: The null hypothesis is that $\mathrm{P}\left(\mathrm{C}>\mathrm{c}^{\prime}\right)<=.05$.

Question: This leads to the next related question regarding a comparison between the two types of errors. As an example, the errors are defined as:

Error a: Declare that in a sampled population the proportion of cylinders with concentration of a given TRU is higher than $c^{\prime}$ is more than (a given value) $p$ when in fact it is less than $p$.

Error b: Declare that in a sampled population the proportion of cylinders with concentration of a given TRU is higher than $c^{\prime}$ is less than (a given value) $p$ when in fact it is greater than $\mathrm{p}$.

Response: Loosely speaking, in (a) we are overestimating the proportion $\mathrm{p}$ of highly contaminated cylinders and underestimating it in (b). Which of the two errors is more serious? Based on the statement of null and alternate hypotheses in the proposal it is concluded that "error a" is the more serious error. But it does not elaborate the reasons. 
Question: Which of the two errors is more serious in our context so that its occurrence can be avoided if possible with more certainty? (It happens with a low probability that can be limited by the level of the test built in the design.)

Response: The question of which type of error is more serious is not addressed here. Because the context is low levels of contamination at a contamination treatment facility, it is not clear which error type would be more serious.

Question: In Appendix C (pg. 49, paragraph 2), you let $Z$ denote the maximum concentration in a set of either ten or twenty cylinder samples. With $\mathrm{Z}$ being the maximum in a random sample, it is itself random. This makes the quantity $\mathrm{P}(\mathrm{C}>$ $\mathrm{Z}$ ) random also. The classical framework of inference regarding parameters is no longer applicable.

Response: The maximum concentration $\mathrm{Z}$ is likely to be a detection limit, or if uncensored readings are reported, they will likely be below an effective detection limit. In either case, $Z$ will not be random, at least not in a way that has much, if any, bearing on actual concentrations. If the data is uncensored and above detection limits, then the classical framework of inference regarding parameters does not apply, strictly speaking, but we are proposing it as an approximation. I will add a paragraph to this effect to the text. I do not know how to correct for the randomness of $\mathrm{Z}$ or a better approach to calculating a $\mathrm{UCB}$ for $\mathrm{P}\left(\mathrm{C}>\mathrm{c}^{\prime}\right)$.

Question: In Appendix C (pg. 49), you discuss the possibility of the function P $(\mathrm{C}>\mathrm{c})$ being sigmoidal. Is there a purity or composition specification on reactor return material used as feed material at the GDPs? If so, are $\mathrm{Np}, \mathrm{Pu}, \mathrm{Am}$, and Tc specified or certified to be below a certain level? If so, the theoretical shape of $\mathrm{P}(\mathrm{C}>\mathrm{c})$ would be a step or, more likely, a very steep sigmoidal shape. This would argue in favor of your Bound discussions and (Bound-AL) would not be much greater than (AL-Z). If not, then the relative values of (Bound-AL) and (AL-Z) are unknown and conservative estimates based on processing knowledge must be made. Is your example (Bound-AL) = 2 (AL-Z) based on any such knowledge?

Response: The answer to all of the questions is no. We did not use this approach because we could not answer these questions. 


\section{Appendix C: History of Tails Cylinder Number 137128 \\ (Letter to Peer Review Team from Bob Hightower, ORNL)}

Here is a quick summary of what has been found so far on the history of cylinder 137128. This information was obtained from cylinder records kept at Kevil and from the USEC data base. Additional research is going to be necessary to gain more details on the feeding of the cylinder.

It appears that this cylinder may have been cold fed into the cascade and there could have been some operational problems encountered. The cylinder was at the C-337-A Feed Facility for approximately 15 months. Just as a note I have been told by operations personnel that the rate of feed could effect what constituents remain in the heel of the cylinder. Cylinder Number 137128 is a 14-ton Model G 48-inch cylinder that was manufactured by Modern Welding Co. Inc of Owensboro Kentucky in 1989. The cylinder was filled with $0.4506 \%$ assay depleted material at the C-315 Tails Withdrawal Facility during the period between June 28, 1989 and July 10, 1989. (This was the only time this cylinder was filled.) The cylinder was placed in storage in the C-745-K Cylinder Storage Yard on July 10, 1989. On July 21, 1989 the cylinder was relocated to the C-337-A Feed Facility. During the period from July 21, 1989 to October 31, 1990 the cylinder was partially fed into the cascade from a gross weight of 30,592 lbs. down to 11,120 lbs. On October 31, 1990/November 1, 1990 the cylinder was fed into the cascade down to a $45 \mathrm{lbs}$. heel quantity. On November 1, 1990 the cylinder was relocated to the C-745-C Cylinder Storage Yard. The cylinder was washed at the C-400 Wash Facility during the period between December 2 and 20, 1999.

Quality Evaluation records dated July 14 and 261989 indicate that the cylinder was damaged while at PGDP and had a gouge (0.145-inch deep $\times 1.25$-inches long x 0.5-inch wide) on the valve-end head. The QE Report recommended that the cylinder be cold fed.

-Bob Hightower 